\title{
Clinical profile and outcome of septic shock in children admitted to a tertiary care referral hospital
}

\author{
Kurade $\mathbf{A}^{1}$, Dhanawade $\mathbf{S}^{2}$ \\ ${ }^{1}$ Dr Aditya Kurade, Resident, ${ }^{2}$ Dr Sara Dhanawade, Professor, Both are affiliated with Department of Pediatrics, Bharati \\ Vidyapeeth Deemed University Medical College \& Hospital, Sangli, Maharashtra, India
}

Address for Correspondence: Dr Aditya Kurade, Resident, Department of pediatrics Bharati Vidyapeeth Deemed University Medical College \& Hospital, Sangli. E-mail: aditya_kurade@yahoo.com

\begin{abstract}
Background: There is dearth of studies on detailed clinical profile and outcome of pediatric septic shock in India. Objective: To describe clinical profile and outcome of septic shock in children. Methods: This retrospective study was conducted from June 2010 to June 2013 in a tertiary care hospital. Case records of children aged 1month to 18 years with diagnosis of septic shock were analyzed. Results: A total of 94(9\%) out of 1035 admissions had shock and 53(56.3\%) of them had a diagnosis of septic shock. Analysis was done on 43 (M:F, 20:23). The mean age was 3 year (range 1month14 years). Maximum (48.83\%) cases were in infancy. The common presenting symptom was fever $(62.79 \%)$ followed by altered mental status in 30.23\%. Pediatric SIRS criteria was met in 35(81.3\%). Most common abnormal lab parameters were elevated liver enzymes $(86.04 \%)$ followed by anemia $(62.79 \%)$ and leukocytosis $(60.46 \%)$. Thrombocytopenia and Coagulopathy was seen in $55.81 \%$ and $60.41 \%$ respectively. Pneumonia was the commonest etiology (51.1\%) followed by cellulitis/abscess in $30.2 \%$. Blood culture was positive in $18.6 \%$ and Staphylococcus was the commonest organism. Frequency of MODS was $90.69 \%$. Most (74.41\%) cases presented with decompensated shock and $97.67 \%$ required inotropes. Majority (88.37\%) required mechanical ventilation. The mortality rate was $60.46 \%$ and mean duration of PICU was 8.3 days. Anemia, leucopenia, decompensated shock and need for mechanical ventilation were significantly associated with mortality $(\mathrm{p}<0.05)$. Conclusion: Septic shock was the most common type of shock encountered in PICU and carries a high mortality. Maximum numbers of patients were below 1year and pneumonia was the commonest underlying cause.
\end{abstract}

Keywords: Clinical Profile, Incidence, Septic shock, Mortality, SIRS.

\section{Introduction}

Sepsis is one of the most common causes of morbidity and mortality in infants and children worldwide particularly in developing countries [1]. The global data on sepsis in children are incomplete but it is estimated that infection accounts for more than $60 \%$ mortality under five children. According to World Health Organization (WHO) four big causes of death in children worldwide are severe Pneumonia (1.9 million deaths/year), severe diarrhea (1.6 million/year), severe malaria (1.1 million/year), severe measles $(5,50,000$ deaths/year). World Health Organization (WHO) used the term severe when children develop acidosis or hypotension or both [2]. Sepsis causes a release of inflammatory mediators,maldistribution of intravascular

Manuscript received: $12^{\text {th }}$ March 2016 Reviewed: $25^{\text {th }}$ March 2016

Author Corrected; $8^{\text {th }}$ April 2016

Accepted for Publication: 20 th April 2016 volume and depression of myocardial function resulting into septic shock. Septic shock is a clinical picture arising out of tissue hypoperfusion following microbial infection. The worldwide burden of pediatric septic shock is huge. Estimates of the incidence of sepsis suggest that there are more than 42,000 cases annually in the United States and millions worldwide and 5-30\% of total sepsis patients develop septic shock [3]. The mortality rate in septic shock patients may be as high as $50 \%$. The outcome in septic shock is worse when associated with co-morbidities and multiple organ dysfunctions [3].

Advances in pediatric intensive care guidelines for septic shock management improved survival of children overall. However septic shock continues to be an important cause of morbidity and mortality in the 
resource limited countries like India [1]. In 2001, a study was published on treatment of sepsis, which stated the importance of early recognition and treatment. In this study mortality rate was reduced by $16 \%$ by using a form of bundled care called early goal directed therapy. This early directed goal therapy consists of set of interventions based on evidence based practice [4]. Early directed goal therapy was adopted by surviving sepsis campaign to improve diagnosis, management and survival of patient with sepsis [4].

Patients presenting with sepsis or septic shock progress rapidly to serious states, and if left untreated, may rapidly progress to death. Even with treatment the mortality in septic shock may be as high as $50 \%$. It is estimated that sepsis and septic shock is the $4^{\text {th }}$ leading cause of hospital admission [5]. The severity and high mortality of this condition highlights that it is a major challenge in front of health care system. There is paucity of data on the epidemiology of shock in developing countries. Studies on pediatric septic shock from India are limited. This study was undertaken to analyze the clinical profile and outcome of septic shock in a tertiary care teaching hospital.

\section{Material \& Methods}

This retrospective study was conducted in pediatric intensive care unit (PICU) of a tertiary care teaching hospital in western Maharashtra from June 2010-June 2013 (3 years). Our PICU is a referral center with 8 beds catering to districts in western Maharashtra and neighboring Karnataka. The unit has an average of 400 admissions per year.

A database of all admission to PICU during the study period was queried. Records of all children aged 1 month to 18 years with shock were reviewed. Those subjects who fulfilled the diagnostic criteria of septic shock on admission or who developed shock during hospital admission were included in the study.

Septic shock was defined as clinical suspicion presenting with hypothermia or hyperthermia accompanied by hypotension and / alteration in perfusion. Diagnosis of Severe inflammatory response syndrome (SIRS), sepsis and MODS were defined as per established criteria [6, 7]. Subjects whose records were incomplete or missing or who died within 4 hours were excluded. The demographic profile, clinical presentation, laboratory parameters and outcome were recorded. Details regarding organ dysfunction need for inotropes and mechanical ventilation were also obtained.

Statistical analysis: Continuous variables were summarized using means, while categorical variables were summarized using percentages. The Chi-square test was used to determine the association of different variables in survivors and non-survivors.

\section{Results}

Out of 1035 admissions to PICU during the study period 94 (9\%) cases had shock. Hypovolemic and cardiogenic shock were seen in $37(39.36 \%)$ and $4(4.25 \%)$ cases respectively. Clinical diagnosis of septic shock was made in 53 (56.3\%) out of 94 patients. Total of 43 cases ( 20 males, 23 females) were included in the final analysis after exclusion of 10 cases ( 4 case record not traceable, 4 incomplete charts, 2 died within 4 hours). The mean age was 3 year (range 1 month - 14 years). Maximum (48.83\%) children were in the age group of $1-12$ months.

The common presenting symptom was fever in $27(62.79 \%)$ cases followed by altered mental status in $13(30.23 \%)$ cases. Other presenting feature were breathlessness in $23.25 \%$, cough in $18.60 \%$, vomiting and abdominal distension in $9.3 \%$ each (Table I). Mean duration of symptoms was 6.4 days.

Table I: Presenting Symptoms.

\begin{tabular}{|l|l|l|}
\hline Presenting symptoms & Number of patients & Percentage \\
\hline Fever & 27 & $62.79 \%$ \\
\hline Altered Mental status & 13 & $30.23 \%$ \\
\hline Breathlessness & 10 & $23.25 \%$ \\
\hline Cough & 8 & $18.60 \%$ \\
\hline Vomiting & 4 & $9.3 \%$ \\
\hline Abdominal distension & 4 & $9.3 \%$ \\
\hline Abdominal Pain & 3 & $6.97 \%$ \\
\hline
\end{tabular}


Table II: SIRS parameters.

\begin{tabular}{|l|l|l|}
\hline SIRS parameters & Number of Patients & Percentage \\
\hline Temperature & 27 & $62.79 \%$ \\
\hline TLC & 36 & $83.72 \%$ \\
\hline HR & 32 & $74.41 \%$ \\
\hline RR & 35 & $81.39 \%$ \\
\hline
\end{tabular}

Table III: Lab parameters.

\begin{tabular}{|l|l|l|}
\hline Parameters & Number of patients & Percentage \\
\hline Anemia $(\mathrm{Hb}<10)$ & 27 & $62.79 \%$ \\
\hline Leucopenia $(\mathrm{TLC}<5000)$ & 6 & $13.95 \%$ \\
\hline Leukocytosis & 26 & $60.46 \%$ \\
\hline Thrombocytopenia $(<1$ lac) & 24 & $55.81 \%$ \\
\hline Coagulopathy & 26 & $60.46 \%$ \\
\hline Hypoglycemia & 10 & $23.25 \%$ \\
\hline Hyperglycemia $(\mathrm{BSL}>150)$ & 14 & $32.55 \%$ \\
\hline Raised liver enzymes & 37 & $86.04 \%$ \\
\hline Base excess $(>5)$ & 19 & $44.18 \%$ \\
\hline
\end{tabular}

Table IV: Comparison between survivors and Non-survivors.

\begin{tabular}{|c|c|c|c|c|c|c|}
\hline \multicolumn{2}{|l|}{ Characteristic } & $\begin{array}{l}\text { Non-survivors } \\
(\mathrm{n}-26)\end{array}$ & $\begin{array}{l}\text { Survivors } \\
\text { (n-17) }\end{array}$ & $\begin{array}{l}\text { Chi- } \\
\text { square }\end{array}$ & p-value & Significance \\
\hline \multirow{2}{*}{ AGE } & $<1$ year & 15 & 10 & \multirow[t]{2}{*}{0.005} & \multirow[t]{2}{*}{0.941} & \multirow[t]{2}{*}{ Not significant } \\
\hline & $>1$ year & 11 & 7 & & & \\
\hline \multirow{2}{*}{ SEX } & Male & 12 & 8 & \multirow[t]{2}{*}{0.003} & \multirow[t]{2}{*}{0.953} & \multirow[t]{2}{*}{ Not significant } \\
\hline & Female & 14 & 9 & & & \\
\hline \multirow{2}{*}{ SHOCK } & Compensated & 3 & 8 & \multirow[t]{2}{*}{6.812} & \multirow[t]{2}{*}{0.009} & \multirow[t]{2}{*}{ Highly significant } \\
\hline & Decompensated & 23 & 9 & & & \\
\hline \multirow{9}{*}{$\begin{array}{l}\text { LAB } \\
\text { PARAMETERS }\end{array}$} & Anemia & 20 & 7 & 5.621 & 0.017 & Significant \\
\hline & Leucopenia & 5 & 1 & 5.596 & 0.018 & Significant \\
\hline & Leukocytosis & 18 & 8 & 2.113 & 0.145 & Not significant \\
\hline & Thrombocytopenia & 16 & 8 & 0.873 & 0.349 & Not significant \\
\hline & Coagulopathy & 14 & 12 & 1.205 & 0.272 & Not significant \\
\hline & Hypoglycemia & 6 & 4 & 0.001 & 0.972 & Not significant \\
\hline & Hyperglycemia & 10 & 4 & 1.043 & 0.306 & Not significant \\
\hline & Liver dysfunction & 23 & 14 & 0.319 & 0.571 & Not significant \\
\hline & Base excess & 12 & 7 & 0.103 & 0.747 & Not significant \\
\hline \multirow{2}{*}{ MODS } & $2-4$ organs & 18 & 14 & \multirow[t]{2}{*}{2.106} & \multirow[t]{2}{*}{0.146} & \multirow[t]{2}{*}{ Not significant } \\
\hline & $>4$ organs & 6 & 1 & & & \\
\hline \multicolumn{2}{|c|}{ INOTROPE required } & 26 & 16 & 1.565 & 0.210 & Not significant \\
\hline \multicolumn{2}{|c|}{$\begin{array}{l}\text { MECHANICAL VENTILATION } \\
\text { required }\end{array}$} & 26 & 12 & 8.653 & 0.003 & Significant \\
\hline
\end{tabular}

Pediatric SIRS criteria were met in 35 (81.3\%) children with septic shock. Abnormal total leukocyte count and tachypnea were the most commonly observed parameters among the SIRS criteria (Table II). Most common hematological abnormalities observed were anemia ( $\mathrm{Hb}<10 \mathrm{gm} \%)$ in 27 (62.79\%) and leukocytosis in 26 (60.46\%). Thrombocytopenia (Platelet $<1 \mathrm{lac}$ ) and Coagulopathy were seen in $55.81 \%$ and $60.41 \%$ respectively. Leucopenia was present only in $13.95 \%$ cases. Mean leukocyte count was 15212 (range 1200 - 39900). Other common biochemical 
derangements included elevated liver enzymes in $86.04 \%$, hyperglycemia in $14(32.55 \%)$, hypoglycemia in $10(23.25 \%)$ and deranged renal function tests in 6 (13.95\%) (Table III). Pneumonia was the commonest cause (51.1\%) followed by cellulitis/abscess in 30.2\%. Other causes included meningitis $(9.3 \%)$, intra-abdominal and urinary tract infections $(4.65 \%)$ each.

Blood culture was positive only in $8(18.6 \%)$ cases and coagulase positive Staphylococcus aureus was the commonest organism. Other organisms isolated were Pseudomonas, Acinetobacter and Enterococcus. Frequency of MODS was $90.69 \%$ and $32.55 \%$ had 4 or more organs involved. Majority (74.41\%) cases presented with decompensated shock. Most $(97.67 \%)$ patients were fluid refractory and inotrope dependent. Majority (88.37\%) required mechanical ventilation. The mortality rate was $60.46 \%$. Mean duration of PICU was 8.3 days (range 8 hours - 36 days). On comparing various clinical and laboratory parameters between survivors and non-survivors it was observed that mortality was significantly associated with anemia, leucopenia, decompensated shock and mechanical ventilation (Table IV).

\section{Discussion}

The frequency of shock in intensive care was $9 \%$ in this study. Septic shock was the most common type of shock followed by hypovolemic shock. According to western data shock occurs in $2 \%$ of hospitalized children while one Indian study reported frequency of $4.3 \%$ [8]. However there are no such data regarding incidence of shock in developing countries. Age distribution showed that nearly half $(46.52 \%)$ of the patients were below one year. There was no gender related difference in the occurrence of septic shock. Our findings are consistent with the previous studies $[2,5,8$, 9].

Fever $(62.79 \%)$ was the most common presenting symptom followed by altered mental status (30.23\%). Other symptoms such as breathlessness and cough were seen in $23.25 \%$ and $18.60 \%$ respectively. A study from Libya reported fever (88\%) as the most common presenting complaint followed by cough (38\%), diarrhea (35\%) and vomiting (28\%) [9]. A Study from Romania also reported that most children (98\%) presented with changes in core temperature followed by significant changes in heart rate and tachypnea [5]. Majority $(81.3 \%)$ of patients diagnosed with septic shock in the present study met the SIRS criteria.

Total leukocyte count and temperature were the most common abnormalities observed in subjects fulfilling SIRS criteria. Militaru $M$ et al observed that most (56\%) patients fulfilled 3 out of 4 criteria of SIRS with most common abnormal criteria was temperature followed by leukocyte count [5].

Children with septic shock were found to present with a variety of abnormal laboratory parameters. Most common hematological abnormalities observed were anemia $(62.79 \%)$ followed by leukocytosis $(60.46 \%)$ and thrombocytopenia (55.81\%). Elevated liver enzyme was the most (86.04\%) common biochemical abnormality seen in this study. In a study from Libya, Benamer et al observed leukocytosis in 50\%, anemia in $40 \%$, leucopenia in $10 \%$ and raised liver enzymes in $43 \%$. They observed thrombocytosis (38\%) more frequently than thrombocytopenia (10\%) [9]. As compared to the above study higher proportion of children in the present study had alteration in laboratory variables. Serum lactate and ionic calcium were not done in all patients during study period hence was not included in the analysis.

Pneumonia was the most common cause of septic shock in our study. Fisher JD et al and Gaines NN et al also reported predominance of respiratory illness and isolation of respiratory pathogen from the septic shock patients $[10,11]$. A study from Romania also reported respiratory infection to be the most (64\%) common etiology followed by digestive tract infection and urinary tract infection [5].

Blood culture yield in the present study was low (18.6\%) and Staphylococcus was the most common organism isolated. Low yield of blood culture has been reported by earlier authors $(5,8)$. However some studies have reported higher culture positivity with predominance of gram negative organisms [12, 13, 14]. Low blood culture yield in the present study can be explained by the fact that most of our patients were referred and had prior antibiotic treatment.

Majority $(74.41 \%)$ patients presented with hypotensive shock. They were fluid refractory and inotrope dependent. This clearly reflects the existing pattern of late referrals and lack of awareness among the health professionals. Most (90.69\%) patients had multiple organ dysfunction and $32.55 \%$ patients had 4 or more organ involvement. Similar observation is reported in a 
previous study [5]. High mortality (60.46\%) was observed in the present study. The literature reports high mortality rate of over $50 \%$ in pediatric patients with septic shock [14]. An Indian study from PGI, Chandigarh also reported high $(65.8 \%)$ mortality rate in fluid refractory septic shock [15]. Other Indian studies have reported morality rates of $47 \%$ from Punjab, $50 \%$ from AIIMS, Delhi, 58\% from Rohtak, Haryana [8, 16, 17]. Our findings are in line with other Indian studies. A study from Romania also reported mortality rate of 53\% [5].

We compared various clinical and laboratory variables between survivors and non-survivors. Among the lab parameters anemia and leucopenia had significant association with mortality. Decompensated shock and need for mechanical ventilation were also significantly associated with mortality. Han YY et al reported that non-survivors were treated with more inotropic therapies as compared to survivors [18]. In a recent study from Rohtak where authors evaluated the hemodynamic and laboratory parameters which discriminated survivors from non survivors, it was found that mortality was not predicted by any individual factor like time lag to PICU transfer, duration of PICU stay, presence of MODS and PRISM score at admission [17]. However this study was limited by the small sample size.

Early detection and reversal of shock improves rate of survival [19]. This could be one of the main reasons for high mortality in our study as most of patients presented in decompensated shock. Interventional study had been done in rural India states the importance of early detection and intervention in which health-care workers gave 5 days course of oral co-trimoxazole and intramuscular gentamicin to neonate with signs of sepsis. This study found decreased mortality rate from $16 \%$ to $3 \%$ with early intervention [20]. Han YY et al also reported reversal of shock at a median time of 75 minutes associated with $96 \%$ survival and more than 9 fold increased odds of survival while each additional hour with persistent shock was associated with more than 2 fold increased odds of mortality [18]. Management of septic shock largely depends on early recognition, infrastructure availability, presence of committed staff and adherence to the management guidelines. Surviving sepsis campaign guidelines have been derived from developed countries and there is a need to have more simpler and practical guidelines for resource limited countries. Retrospective nature was the major limitation of this study. Variation in filling the patient records, completeness of data as well as variation in the management protocol could have influenced our findings. Adherence to early goal directed therapy could not be evaluated because of the study nature.

\section{Conclusion}

Septic shock in Indian children is associated with high mortality. Children below 1 year are the most vulnerable group and pneumonia is the commonest underlying cause. Early recognition, timely referral and aggressive treatment are critical in reducing the mortality. It is desirable to have customized protocol for each unit in line with surviving sepsis campaign guidelines. Larger well designed studies using uniform protocols are needed to study the epidemiology and outcome of septic shock in Indian children.

\section{Contribution-}

AK: Data collection, statistical analysis, literature search and manuscript preparation.

SD: Study concept and critical revision of manuscript.

Source of Support: Nil

Conflict of Interest: None

Permission of IRB: Yes

\section{References}

1. Khilnani P, Singhi S, Lodha R, Santhanam I, Sachdev A, Chugh K, Jaishree M, Ranjit S, Ramachandran B, Ali U, Udani S, Uttam R, Deopujari S. Pediatric Sepsis Guidelines: Summary for resourcelimited countries. Indian J Crit Care Med. 2010 Jan;14(1):41-52. doi: 10.4103/0972-5229.63029.

2. Watson RS, Carcillo JA. Scope and epidemiology of pediatric sepsis. Pediatr Crit Care Med. 2005 May; 6 (3 Suppl): S3-5.

3. Kutko MC, Calarco MP, Flaherty MB, Helmrich RF, Ushay HM, Pon S, Greenwald BM. Mortality rates in pediatric septic shock with and without multiple organ system failure. Pediatr Crit Care Med. 2003 Jul;4(3):333-7.

4. Dellinger R, Levy M, Rhodes A, Annnae D, Gerlach $\mathrm{H}$, Opal $\mathrm{S}$ et al. Surviving sepsis campaign: international guidelines for management of severe sepsis and septic shock. Critical care medicine 2012; 41: 580-637.

5. Militaru M, Martinovici D. Our experience in paediatric sepsis. Jurnalul pediatrului 2005; 8: 26-31. 
6. Goldstein B, Giroir B, Adrienne R. International pediatric sepsis consensus conference: Definitions for sepsis and organ dysfunction in pediatric. Pediatr Crit Care Med 2005; 6: 2-5.

7. Surviving Sepsis Campaign: International guidelines for management of severe sepsis and septic shock. Crit Care Med 2008; 36: 861-3.

8. Singh D, Chopra A, Pooni PA, Bhatia RC. A clinical profile of shock in children in Punjab, India. Indian Pediatr. 2006 Jul;43(7):619-23.

9. Benamer HM, Alsaiti AA, Bofarraj M, Abud H, Tip RM. Diagnosis, management and outcome of sepsis at Benghazi children hospital(1 year review). Pediat Therapeut 5: 267. Doi:10.4172/2161-0665.1000267.

10. Fisher JD, Nelson DG, Beyersdorf H, Satkowiak LJ. Clinical spectrum of shock in the pediatric emergency department. Pediatr Emerg Care. 2010 Sep;26(9):622-5. doi: 10.1097/PEC.0b013e3181ef04b9.

11. Gaines NN, Patel B, Williams EA, Cruz AT. Etiologies of septic shock in a pediatric emergency department population. Pediatr Infect Dis J. 2012 Nov;31(11):1203-5. doi: 10.1097/INF.0b013e3182678ca9.

12. Dahmash NS, Chowdhury NH, Fayed DF. Septic shock in critically ill patients: aetiology, management and outcome. J Infect. 1993 Mar;26(2):159-70.

13. Contran RS, Kumar V, Robbins SL. Shock in fluid and hemodynamic derangements. Robins Pathologic Basis of disease. 114-9.
14. Pollack MM, Fields AI, Ruttimann UE. Sequential cardiopulmonary variables of infants and children in septic shock. Crit Care Med. 1984 Jul;12(7):554-9.

15. Valoor HT, Singhi S, Jayashree M. Low-dose hydrocortisone in pediatric septic shock: an exploratory study in a third world setting. Pediatr Crit Care Med. 2009 Jan;10(1):121-5. doi: 10.1097/PCC.0b013e3181936ab3.

16. Sarthi M, Lodha R, Vivekanandhan S, Arora NK. Adrenal status in children with septic shock using lowdose stimulation test. Pediatr Crit Care Med. 2007 Jan;8(1):23-8.

17. Kaur G, Vinayak N, Mittal K, Kaushik JS, Aamir M. Clinical outcome and predictors of mortality in children with sepsis, severe sepsis, and septic shock from Rohtak, Haryana: A prospective observational study. Indian J Crit Care Med. 2014 Jul;18(7):437-41. doi: 10.4103/0972-5229.136072.

18.Han YY, Carcillo JA, Dragotta MA, Bills DM, Watson RS, Westerman ME, Orr RA. Early reversal of pediatric-neonatal septic shock by community physicians is associated with improved outcome. Pediatrics. 2003 Oct;112(4):793-9.

19. Biban P, Gaffuri M, Spaggiari S, Zaglia F, Serra A, Santuz P. Early recognition and management of septic shock in children. Pediatr Rep. 2012 Jan 2;4(1):e13. doi: 10.4081/pr.2012.e13. Epub 2012 Mar 26.

20. Bang AT, Bang RA, Baitule SB, Reddy MH, Deshmukh MD. Effect of home-based neonatal care and management of sepsis on neonatal mortality: field trial in rural India. Lancet. 1999 Dec 4;354(9194):1955-61.

\section{How to cite this article?}

Kurade A, Dhanawade S, Clinical profile and outcome of septic shock in children admitted to a tertiary care referral hospital: Int J Pediatr Res 2016;3(4):228-233.doi:10.17511/ijpr.2016.i04.04. 